\title{
Educación para la paz como herramienta para construir la cultura de paz
}

\author{
Emmanuel Roldán Loyola*
}

Cabello-Tijerina, P., \& Vázquez-Gutiérrez, R. (2018). Cultura y educación para la paz. Una perspectiva transversal. México: Tirant Lo Blanch.

Ante la violencia como fenómeno de gran preocupación a nivel global, la Organización de la Naciones Unidas para la Educación, la Ciencia y la Cultura (UNESCO, 1989) invitó a todos los Estados y organizaciones a contribuir a la construcción de una nueva concepción de la paz mediante el desarrollo de una cultura de paz. Así, con este objetivo en mente, se llevó a cabo el Congreso Internacional sobre la Paz en la Mente de los Hombres, realizado en Yamusukro (Costa de Marfil), donde se iniciaron una serie de trabajos e iniciativas para construir una cultura de paz.

A pesar de estos esfuerzos, aún en el mundo y, particularmente, en Latinoamérica, los índices de violencia siguen siendo bastante altos. Por ejemplo, según el Índice de Paz Global que elabora el Instituto para la Economía y la Paz (2019), se ha dicho que México se encuentra en la posición 140 de un total de 163 países que lo conforman, además de que es el país menos pacífico de la región de América Central y el Caribe, siendo esta la zona con mayor decrecimiento en el puntaje de dicho índice entre 2018 y 2019.

Con este panorama, retomar los estudios para la paz que nacieron a mediados del siglo XX, así como el concepto de cultura de paz — que es mucho más reciente - es una de las apuestas en este nuevo siglo. Teniendo esto en cuenta, el libro, publicado en México, Cultura y educación para la paz. Una perspectiva transversal, de Cabello-Tijerina y Vázquez-Gutiérrez, ofrece importantes aportaciones por medio de definiciones, conceptos, teorías y metodologías que permitirán llegar a la construcción de una cultura de paz.

Específicamente, esta obra está conformada por seis capítulos a través de los cuales los autores exponen de manera clara y concisa gran parte del conocimiento acumulado sobre la cultura de paz y la educación para la paz.
Así, en el primer capítulo se hace referencia a la ciencia que estudia la paz, la irenología, y a lo largo de este se pueden encontrar el origen de dicha ciencia y las tres tendencias teóricas de la investigación sobre la paz. Además, los autores presentan la definición de Johan Galtung de la investigación para la paz, entendiéndose como la ciencia que "estudia las condiciones que permiten a la sociedad pasar de una situación caracterizada por la institución social de la guerra a una situación de paz" (p. 27). Por otra parte, cabe resaltar dos ideas fundamentales en este capítulo: la primera, que para romper el círculo vicioso de las culturas y estructuras violentas es necesario anteponer una cultura y una estructura de paz donde existan los mecanismos necesarios para solventar los conflictos por medios no violentos; y la segunda, que para pasar de la guerra a la paz se requiere una transición de una sociedad dominada por el Estado a una sociedad civil más activa en la que las personas trabajen, creen y desarrollen el cambio cultural de la violencia hacia la instauración de una cultura de paz.

Asimismo, en el segundo capítulo se plantean las diferentes definiciones, elementos y tipologías de la paz. En particular, en este se explica la importante evolución del concepto, donde el año 1969 resulta de bastante relevancia para la conceptualización de la paz, ya que antes de este era definida como la ausencia de guerra (paz negativa), pero en ese momento Galtung planteó el concepto de paz positiva para poder implantar una cultura de paz, además de que se dotó al concepto de herramientas y de una teoría más amplia para poder estudiarlo.

Por otra parte, en el tercer capítulo se hace énfasis en la cultura de paz, y para ello los autores presentan definiciones, así como elementos y estrategias para su construcción. Cabe destacar que aquí se proponen dos formas para lograr la construcción de una cultura de paz: la primera, a través de microestrategias, es decir, acciones positivas que contribuyen a generar pequeños cambios en nuestras costumbres $\mathrm{y}$ estilos de vida, donde la educación es fundamental; y

\footnotetext{
* Analista del Observatorio Ciudadano de Seguridad y Paz, Consejo Ciudadano de Seguridad y Justicia del estado de Puebla. emmanuuel.16@hotmail.com
} 
370

la segunda, mediante macroestrategias, entendidas como programas, declaraciones y acciones internacionales que finalmente tienen impacto en las distintas poblaciones en donde se aplican dichas estrategias.

Adicional a esto, los autores dedican el cuarto capítulo a la educación para la paz, y en él plasman los cambios de la educación a lo largo de la historia, con énfasis en las diferencias entre la educación tradicional y la educación moderna. Los autores también explican cómo surgió la dicho concepto, su visón, sus principios y distintas definiciones. Por ejemplo, mencionan que la educación para la paz - para autores como Jares - es un "proceso educativo, dinámico, continuo y permanente, fundamentado en los conceptos de paz positiva y en el conflicto como elemento significantes y definidores, y que, a través de la aplicación de enfoques socioafectivos y problematizantes, pretende desarrollar un nuevo tipo de cultura, la cultura de paz" (p. 88). Es importante mencionar que este capítulo termina con el tema de la educación integral, la cual implica que para lograr las metas pensadas es necesario ampliar los objetivos educativos - que por lo regular se enfocan en el desarrollo intelectual - para dar cabida a otras dimensiones de la persona, como lo son la educación en valores, en habilidades sociales y en resolución de conflictos, y que en estas se tenga como base la tolerancia, el respeto, la cooperación y la convivencia.

Con estas definiciones claras, en el quinto capítulo los autores resaltan la importancia de la labor que juegan las instituciones sociales en la educación integral. Aquí, se da especial importancia a la familia, la escuela y la sociedad como las principales instituciones socializadoras que influyen para que cada persona interiorice comportamientos, normas, actitudes, hábitos y valores desde el nacimiento hasta la edad adulta. En este apartado es de resaltar la sinergia que debe existir entre estas tres instituciones para mejorar la convivencia entre las personas y construir sociedades más pacíficas.

Finalmente, en el último capítulo se habla de la transversalidad práctica de la educación para la paz, haciendo referencia a la introducción de los contenidos de esta en todas las asignaturas curriculares, así como en las prácticas y actividades diarias para lograr la adquisición y la interiorización de la paz. En última instancia, la formación de agentes de paz es el objetivo principal, y esto se logra a través del involucramiento de dichos agentes con las instituciones socializadoras más próximas.

En conclusión, el libro Cultura y educación para la paz. Una perspectiva transversal es ecuánime a la hora de abordar cada uno de sus temas y contribuye teóricamente a la pacificación, tal como los autores se lo propusieron. Esta obra resulta inspiradora y motiva a sus lectores a llevar a la práctica cada una de las propuestas y metodologías allí planteadas, para así contribuir en la construcción de sociedades más pacíficas.

\section{Referencias}

Instituto para la Economía y la Paz. (2019). Global Peace Index. Sidney: Autor. Recuperado de http://visionofhumanity.org/ app/uploads/2019/06/GPI-2019-web003.pdf

UNESCO. (1989). Aplicación de las conclusiones del Congreso Internacional sobre la paz en la mente de los Hombres: Declaración de Yamusukro; Declaración de Sevilla sobre la violencia. Recuperado de https://unesdoc.unesco.org/ ark:/48223/pf0000094314_spa 